\title{
A Space-Mapping Interpolating Surrogate Algorithm for Highly Optimized EM-Based Design of Microwave Devices
}

\author{
John W. Bandler, Fellow, IEEE, Daniel M. Hailu, Student Member, IEEE, Kaj Madsen, and Frank Pedersen
}

\begin{abstract}
We justify and elaborate in detail on a powerful new optimization algorithm that combines space mapping (SM) with a novel output SM. In a handful of fine-model evaluations, it delivers for the first time the accuracy expected from classical direct optimization using sequential linear programming. Our new method employs a space-mapping-based interpolating surrogate (SMIS) framework that aims at locally matching the surrogate with the fine model. Accuracy and convergence properties are demonstrated using a seven-section capacitively loaded impedance transformer. In comparing our algorithm with major minimax optimization algorithms, the SMIS algorithm yields the same minimax solution within an error of $10^{-15}$ as the Hald-Madsen algorithm. A highly optimized six-section $H$-plane waveguide filter design emerges after only four HFSS electromagnetic simulations, excluding necessary Jacobian estimations, using our algorithm with sparse frequency sweeps.
\end{abstract}

Index Terms-Computer-aided design (CAD) algorithms, electromagnetics, filter design, interpolating surrogate, microwave modeling, optimization, output space mapping (OSM), space mapping (SM), surrogate modeling.

\section{INTRODUCTION}

$\mathbf{E}$ LECTROMAGNETIC (EM) simulators, long used by engineers for design verification, need to be exploited in the optimization process. However, the higher the fidelity (accuracy) of the EM simulations, the more expensive direct optimization becomes. For complex problems, EM direct optimization may be prohibitive. Space mapping (SM) [1] aims to combine the speed and maturity of circuit simulators with the accuracy of EM solvers. The SM concept exploits "coarse" models (usually computationally fast circuit-based models) to construct a surrogate that is faster than the "fine" models (typically CPUintensive full-wave EM simulations) and at least as accurate as the underlying "coarse" model [1]-[4]. The surrogate is

Manuscript received April 29, 2004; revised July 8, 2004. This work was supported in part by the Natural Sciences and Engineering Research Council of Canada under Grant OGP0007239 and Grant STPGP 269760, through the Micronet Network of Centres of Excellence and Bandler Corporation.

J. W. Bandler is with the Simulation Optimization Systems Research Laboratory, Department of Electrical and Computer Engineering, McMaster University, Hamilton, ON, Canada L8S 4K1 and also with Bandler Corporation, Dundas, ON, Canada L9H 5E7 (e-mail: bandler@mcmaster.ca).

D. M. Hailu is with the Simulation Optimization Systems Research Laboratory, Department of Electrical and Computer Engineering, McMaster University, Hamilton, ON, Canada L8S 4K1.

K. Madsen and F. Pedersen are with the Department of Informatics and Mathematical Modelling, Technical University of Denmark, DK-2800, Lyngby, Denmark.

Digital Object Identifier 10.1109/TMTT.2004.837197 iteratively updated by the SM approach to better approximate the corresponding fine model.

From the mathematical motivation of SM [4], it was found that SM-based surrogate models provide a good approximation over a large region, whereas the first-order Taylor model is better close to the optimal fine-model solution. Based on this finding and an explanation of residual misalignment, Bandler et al. [5] proposed the novel output space mapping (OSM) to further correct residual misalignment close to the optimal fine-model solution. OSM reduces the number of computationally expensive fine-model evaluations, while improving accuracy of the SM-based surrogate.

This paper elaborates on a new SM algorithm. Highly accurate space-mapping interpolating surrogate (SMIS) models are built for use in gradient-based optimization [6]. The SMIS is required to match both the responses and derivatives of the fine model within a local region of interest. It employs an output mapping to achieve this.

The SMIS framework is formulated in Section IV. An algorithm based on it is outlined in Section V. Convergence is compared with two classical minimax algorithms, and a hybrid aggressive space-mapping (HASM) surrogate-based optimization algorithm using a seven-section capacitively loaded impedance transformer. Finally, the SMIS algorithm is implemented on a six-section $H$-plane waveguide filter [7].

\section{Design PRoblem}

\section{A. Design Problem}

The original design problem is

$$
\boldsymbol{x}_{f}^{*}=\arg \min _{\boldsymbol{x}_{f}} U\left(\boldsymbol{R}_{f}\left(\boldsymbol{x}_{f}\right)\right) .
$$

Here, $\boldsymbol{R}_{f}: \mathbb{R}^{n} \rightarrow \mathbb{R}^{m}$ is the fine-model response vector, e.g., $\left|S_{11}\right|$ at selected frequency points $\omega_{i}, i=1, \ldots, m, m$ is the number of response sample points, and the fine-model point is denoted $\boldsymbol{x}_{f} \in \mathbb{R}^{n}$, where $n$ is the number of design parameters. $U: \mathbb{R}^{m} \rightarrow \mathbb{R}$ is a suitable objective function, and $\boldsymbol{x}_{f}^{*} \in \mathbb{R}^{n}$ is the optimal design.

\section{OSM}

OSM addresses residual misalignment between the optimal coarse-model response and the true fine-model optimum response $\boldsymbol{R}_{f}\left(\boldsymbol{x}_{f}^{*}\right)$. In the original SM [1], an exact match between 


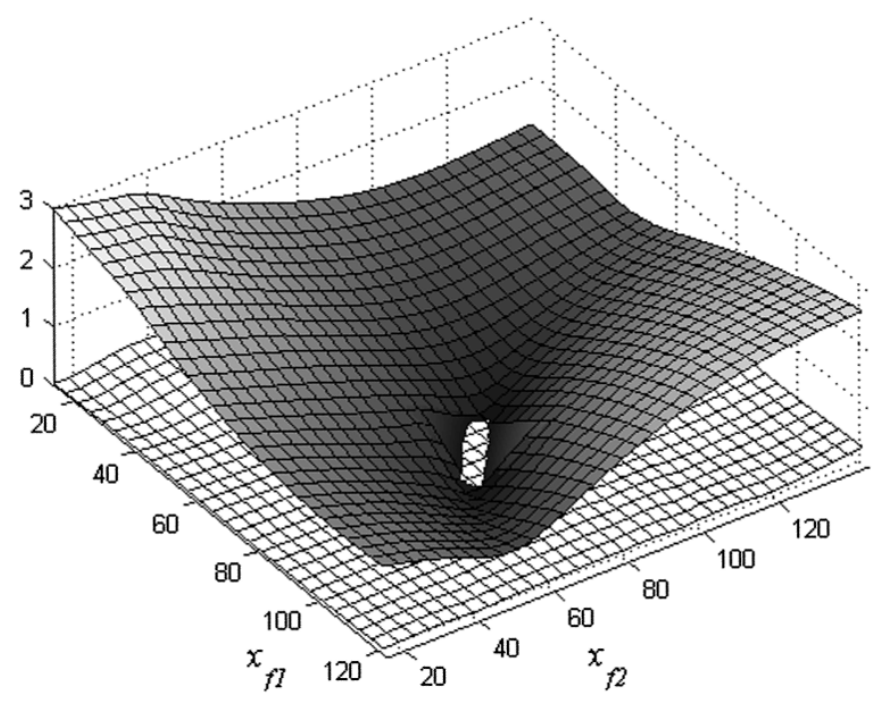

Fig. 1. Error plots for a two-section capacitively loaded impedance transformer [4] exhibiting the quasi-global effectiveness of SM (light grid) versus a classical Taylor approximation (dark grid). See text.

the fine model and mapped coarse model is unlikely. For example, a coarse model such as $R_{c}=x^{2}$ will never match the fine model $R_{f}=x^{2}-2$ around its minimum with any mapping $x_{c}=P\left(x_{f}\right), x_{c}, x_{f} \in \mathbb{R}$. An "output" or response mapping can overcome this deficiency by introducing a transformation of the coarse-model response based on a Taylor approximation [8].

The results of Bakr et al. [4] indicate that "input" SM-based surrogates are good approximations to the fine model over a large region, which makes them useful in the early stages of an optimization process. The residual misalignment between the corresponding mapped coarse model(s) and the fine model renders an exact match between them unlikely. Consequently, convergence to $\boldsymbol{x}_{f}^{*}$ should not be expected.

Fig. 1 depicts model effectiveness plots [4] for a two-section capacitively loaded impedance transformer at the final iterate $\boldsymbol{x}_{f}^{(i)}$, approximately $[74.2379 .27]^{T}$. Centered at $\boldsymbol{h}=\mathbf{0}$, the light grid shows $\left\|\boldsymbol{R}_{f}\left(\boldsymbol{x}_{f}^{(i)}+\boldsymbol{h}\right)-\boldsymbol{R}_{c}\left(\boldsymbol{L}_{p}\left(\boldsymbol{x}_{f}^{(i)}+\boldsymbol{h}\right)\right)\right\|$. This represents the deviation of the mapped coarse model (using the Taylor approximation $\boldsymbol{L}_{p}: \mathbb{R}^{n} \rightarrow \mathbb{R}^{n}$ to the mapping, i.e., a linearized mapping) from the fine model. The dark grid shows $\left\|\boldsymbol{R}_{f}\left(\boldsymbol{x}_{f}^{(i)}+\boldsymbol{h}\right)-\boldsymbol{L}_{f}\left(\boldsymbol{x}_{f}^{(i)}+\boldsymbol{h}\right)\right\|$. This is the deviation of the fine model from its classical Taylor approximation $L_{f}: \mathbb{R}^{n} \rightarrow \mathbb{R}^{m}$. The gradient of the two-section capacitively loaded impedance transformer, used in the Taylor approximation, was obtained analytically using the adjoint network method [9]. The light grid surface passing over the dark grid surface near the center of Fig. 1 verifies that the Taylor approximation is most accurate close to $\boldsymbol{x}_{f}^{(i)}$, whereas the mapped coarse model is best over a larger region. The reason that the Taylor approximation is best in the vicinity of $\boldsymbol{x}_{f}^{(i)}$ is that the Taylor approximation interpolates at the development point, whereas the mapped coarse model does not.

Based on the above finding, Bakr et al. [10] use a surrogate that is a convex combination of a mapped coarse model and a first-order Taylor approximation of the fine model. Madsen and Søndergaard [11] prove convergence of such HASM algorithms.

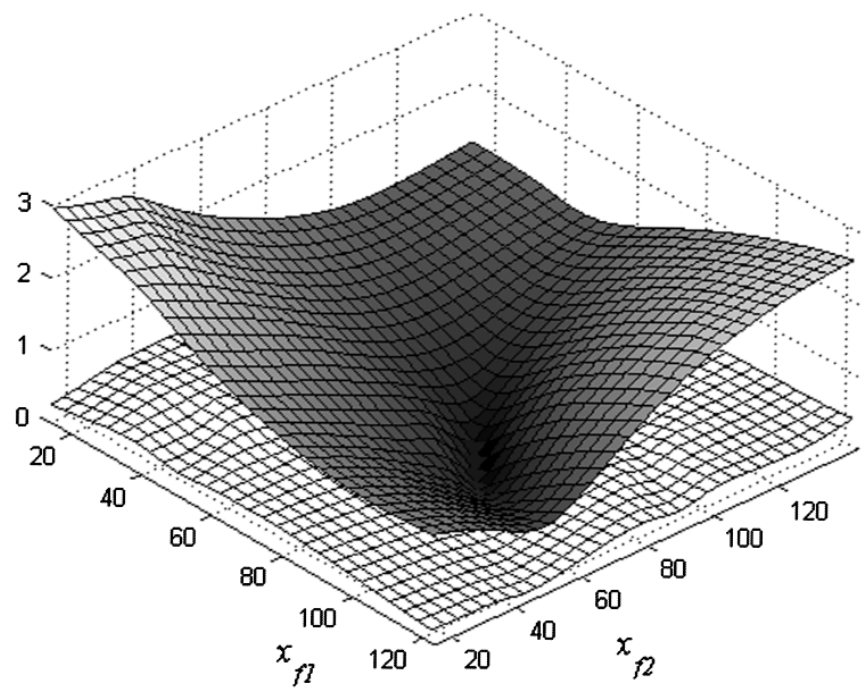

Fig. 2. Error plots for a two-section capacitively loaded impedance transformer [4] exhibiting the quasi-global effectiveness of SM-based interpolating surrogate, which exploits OSM (light grid) versus a classical Taylor approximation (dark grid). See text.

In this paper, we introduce a novel method to ensure convergence of the SM technique. OSM is incorporated into SMIS to ensure that we obtain the same solution as classical direct gradient-based optimization. Fig. 2 depicts model effectiveness plots for the two-section capacitively loaded impedance transformer corresponding to Fig. 1. Centered at $\boldsymbol{h}=\mathbf{0}$, the light grid shows $\left\|\boldsymbol{R}_{f}\left(\boldsymbol{x}_{f}^{(i)}+\boldsymbol{h}\right)-\boldsymbol{R}_{s}\left(\boldsymbol{x}_{f}^{(i)}+\boldsymbol{h}\right)\right\|$. This represents the deviation of the SMIS surrogate from the fine model. The dark grid shows the deviation of the fine model from its classical Taylor approximation as in Fig. 1. Thus, Fig. 2 demonstrates that the SMIS surrogate, because of its interpolating properties, performs better than the first-order Taylor approximation even close to $x_{f}^{(i)}$.

\section{SMIS FRAMEWORK}

\section{A. Surrogate}

The SM-based interpolating surrogate $\boldsymbol{R}_{s}: \mathbb{R}^{n} \rightarrow \mathbb{R}^{m}$ is defined as a transformation of a coarse model $\boldsymbol{R}_{c}: \mathbb{R}^{n} \rightarrow \mathbb{R}^{m}$ through input and output mappings at each sampled response. Fig. 3 illustrates the SMIS framework. Here, $\boldsymbol{P}=\left[\boldsymbol{P}_{1} \ldots \boldsymbol{P}_{m}\right]^{T}$, where $\boldsymbol{P}_{\boldsymbol{i}}: \mathbb{R}^{n} \rightarrow \mathbb{R}^{n}, i=1, \ldots, m$, [1], [2] is an input mapping for the $i$ th coarse response $R_{c, i}$, and $\boldsymbol{O}: \mathbb{R}^{m} \rightarrow \mathbb{R}^{m}$ [8] is an output mapping applied to the coarse response. Using the function $\boldsymbol{R}_{p}: \mathbb{R}^{m \cdot n} \rightarrow \mathbb{R}^{m}$ with individually adjusted coarse responses, defined as $\boldsymbol{R}_{p}\left(\boldsymbol{z}_{1}, \ldots, \boldsymbol{z}_{m}\right)=\left[R_{c, 1}\left(z_{1}\right) \ldots R_{c, m}\left(\boldsymbol{z}_{m}\right)\right]^{T}$, where $z_{i} \in \mathbb{R}^{n}, i=1, \ldots, m$, the surrogate can be expressed as a composed mapping $\boldsymbol{R}_{s}=\boldsymbol{O} \circ \boldsymbol{R}_{p} \circ \boldsymbol{P}$.

We wish to consider individual mappings of each coarse response $R_{c, i}, i=1, \ldots, m$. These (nonlinear) mappings will be approximated by a sequence of local linear mappings. The $i$ th linearized input mapping at the $j$ th iteration is assumed to be of the form

$$
\boldsymbol{P}_{i}\left(\boldsymbol{x}_{f}\right)=\boldsymbol{B}_{i} \boldsymbol{x}_{f}+\boldsymbol{c}_{i}
$$



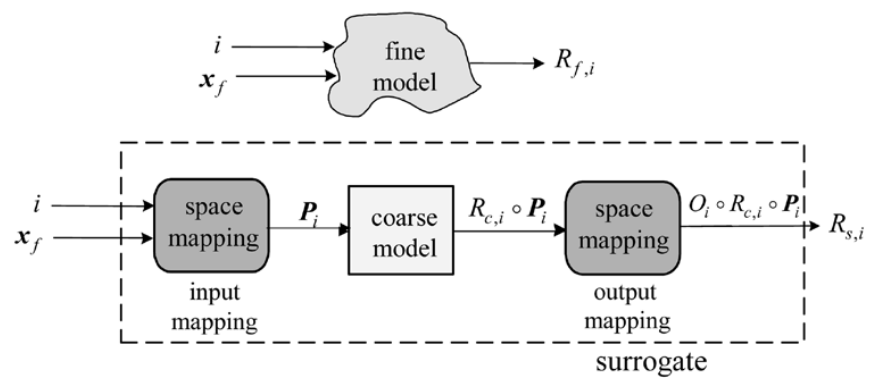

Fig. 3. Illustration of the SMIS concept. The aim is to calibrate the mapped coarse model (the surrogate) to match the fine model using different input and output mappings for each sampled response.

where the matrix $B_{i} \in \mathbb{R}^{n \times n}$ and vector $c_{i} \in \mathbb{R}^{n}$. The $i$ th output mapping is defined as

$$
O_{i}(\boldsymbol{y})=\alpha_{i}\left(y_{i}-\bar{y}_{i}\right)+\beta_{i}
$$

where $y_{i}, \bar{y}_{i}$ are the $i$ th components of $\boldsymbol{y}, \overline{\boldsymbol{y}} \in \mathbb{R}^{m} \cdot \bar{y}_{i}$ is defined as $\bar{y}_{i}=R_{c, i}\left(\boldsymbol{B}_{i} \overline{\boldsymbol{x}}_{f}+\boldsymbol{c}_{i}\right)$, where $\overline{\boldsymbol{x}}_{f}$ is a constant vector. Defining $y_{i}$ similarly, the $i$ th component of the surrogate becomes

$$
\begin{aligned}
R_{s, i}\left(\boldsymbol{x}_{f}\right) & =O_{i}\left(\boldsymbol{R}_{p}\left(\boldsymbol{P}_{i}\left(\boldsymbol{x}_{f}\right)\right)\right) \\
& =\alpha_{i}\left(R_{c, i}\left(\boldsymbol{B}_{i} \boldsymbol{x}_{f}+\boldsymbol{c}_{i}\right)-R_{c, i}\left(\boldsymbol{B}_{i} \overline{\boldsymbol{x}}_{f}+\boldsymbol{c}_{i}\right)\right)+\beta_{i} .
\end{aligned}
$$

We now discuss how to determine the constants $\left\{\boldsymbol{B}_{i}, \boldsymbol{c}_{i}, \alpha_{i}, \beta_{i}\right\}, i=1, \ldots, m$ defining the linear mappings $\boldsymbol{O}$ and $\boldsymbol{P}$. Assume we have reached the $j$ th iterate $\boldsymbol{x}_{f}^{(j)}$ in the iterative search for a solution. At $\boldsymbol{x}_{f}^{(j)}$, the surrogate $\boldsymbol{R}_{s}^{(j)}$ must agree with the fine response [12]

$$
\boldsymbol{R}_{s}^{(j)}\left(\boldsymbol{x}_{f}^{(j)}\right)=\boldsymbol{R}_{f}\left(\boldsymbol{x}_{f}^{(j)}\right) .
$$

We also aim to align the surrogate with the fine-model response at the previous points in the iteration, as well as aim to have agreement between the Jacobians at the current point, i.e.,

$$
\begin{aligned}
\boldsymbol{R}_{s}^{(j)}\left(\boldsymbol{x}_{f}^{(k)}\right) & =\boldsymbol{R}_{f}\left(\boldsymbol{x}_{f}^{(k)}\right), \quad k=1,2, \ldots, j-1 \\
\boldsymbol{J}_{s}^{(j)}\left(\boldsymbol{x}_{f}^{(j)}\right) & =\boldsymbol{J}_{f}\left(\boldsymbol{x}_{f}^{(j)}\right)
\end{aligned}
$$

where $\boldsymbol{J}_{s}^{(j)}\left(\boldsymbol{x}_{f}^{(j)}\right)$ and $\boldsymbol{J}_{f}\left(\boldsymbol{x}_{f}^{(j)}\right)$ are the Jacobians of the surrogate and fine model at $\boldsymbol{x}_{f}^{(j)}$, respectively.

The constants $\left\{\boldsymbol{B}_{i}, \boldsymbol{c}_{i}, \alpha_{i}, \beta_{i}\right\}, i=1, \ldots, m$ are determined in such a way that the alignment (5) holds and the requirements in (6) are satisfied as well as possible (in some sense to be specified). The alignment (5) is satisfied by choosing $\overline{\boldsymbol{x}}_{f}$ and $\beta_{i}$ appropriately. If we let $\overline{\boldsymbol{x}}_{f}=\boldsymbol{x}_{f}^{(j)}$, then (5) only depends on the choice of $\beta_{i}$.

Thus, the $j$ th surrogate of response number $i$ is

$$
\begin{gathered}
R_{s, i}^{(j)}\left(\boldsymbol{x}_{f}\right)=\alpha_{i}^{(j)}\left(R_{c, i}\left(\boldsymbol{P}_{i}^{(j)}\left(\boldsymbol{x}_{f}\right)\right)\right. \\
\left.-R_{c, i}\left(\boldsymbol{P}_{i}^{(j)}\left(\boldsymbol{x}_{f}^{(j)}\right)\right)\right)+\beta_{i}^{(j)}, \\
i=1, \ldots, m \text { and } j=0,1, \ldots
\end{gathered}
$$

where

$$
\boldsymbol{P}_{i}^{(j)}\left(\boldsymbol{x}_{f}\right)=\boldsymbol{B}_{i}^{(j)} \boldsymbol{x}_{f}+\boldsymbol{c}_{i}^{(j)}
$$

In the first iteration, the mapping parameters $\boldsymbol{B}_{i}^{(0)}=\mathbf{I}, \boldsymbol{c}_{i}^{(0)}=$ $0, \alpha_{i}^{(0)}=1$ and $\beta_{i}^{(0)}=\alpha_{i}^{(0)} R_{c, i}\left(\boldsymbol{P}_{i}^{(0)}\left(\boldsymbol{x}_{f}^{(0)}\right)\right)$ are used, which ensure that $\boldsymbol{R}_{s}^{(0)}\left(\boldsymbol{x}_{f}\right)=\boldsymbol{R}_{c}\left(\boldsymbol{x}_{f}\right)$. For $j>0$, the parameter $\beta^{(j)}=R_{f}\left(\boldsymbol{x}_{f}^{(j)}\right)$ is utilized, which ensures (5).

In summary, the surrogate used in the $j$ th iteration is given by

$$
\boldsymbol{R}_{s}^{(j)}\left(\boldsymbol{x}_{f}\right)=\left[R_{s, 1}^{(j)}\left(\boldsymbol{x}_{f}\right) \ldots R_{s, m}^{(j)}\left(\boldsymbol{x}_{f}\right)\right]^{T} .
$$

In each iteration, the surrogate is optimized to find the next iterate by solving

$$
\boldsymbol{x}_{f}^{(j+1)}=\arg \min _{\boldsymbol{x}_{f}} U\left(\boldsymbol{R}_{s}^{(j)}\left(\boldsymbol{x}_{f}\right)\right) .
$$

\section{B. Surface Fitting Approach for Parameter Extraction (PE)}

$\mathrm{PE}$ is a crucial step in any SM algorithm. In this paper, we employ a surface fitting approach for PE, which involves the minimization of residuals between the surrogate and fine models, and extracting the parameters $\boldsymbol{B}_{i}^{(j+1)}, \boldsymbol{c}_{i}^{(j+1)}$, and $\alpha_{i}^{(j)}, i=1, \ldots, m$.

Assume $\boldsymbol{x}_{f}^{(j+1)}$ has been found. We now wish to find the $(j+$ 1)th set of mapping parameters $\left\{\alpha_{i}^{(j+1)}, \boldsymbol{B}_{i}^{(j+1)}, \boldsymbol{c}_{i}^{(j+1)}\right\}$. Since (5) is automatically satisfied by using (7), the aim is to ensure the matching (6). Thus, for finding $\left\{\alpha_{i}^{(j+1)}, \boldsymbol{B}_{i}^{(j+1)}, \boldsymbol{c}_{i}^{(j+1)}\right\}$, we aim to minimize the following set of residuals in some sense [6]:

$$
\begin{aligned}
\boldsymbol{r}_{i}^{(j+1)}(\alpha, \boldsymbol{B}, \boldsymbol{c}) \\
\triangleq\left[\begin{array}{c}
R_{s, i}^{(j+1)}\left(\boldsymbol{x}_{f}^{(1)}, \alpha, \boldsymbol{B}, \boldsymbol{c}\right)-R_{f, i}\left(\boldsymbol{x}_{f}^{(1)}\right) \\
\vdots \\
R_{s, i}^{(j+1)}\left(x_{f}^{(j)}, \alpha, \boldsymbol{B}, \boldsymbol{c}\right)-R_{f, i}\left(\boldsymbol{x}_{f}^{(j)}\right) \\
\boldsymbol{J}_{s, i}^{(j+1)}\left(\boldsymbol{x}_{f}^{(j+1)}, \alpha, \boldsymbol{B}, \boldsymbol{c}\right)-\boldsymbol{J}_{f, i}\left(\boldsymbol{x}_{f}^{(j+1)}\right)
\end{array}\right]
\end{aligned}
$$

where $\boldsymbol{J}_{f, i}$ and $\boldsymbol{J}_{s, i}$ are the $i$ th columns of $\boldsymbol{J}_{f}^{T}$ and $\boldsymbol{J}_{s}^{T}$, respectively. The residual (11) is used during the PE optimization process

$$
\left\{\alpha_{i}^{(j+1)}, \boldsymbol{B}_{i}^{(j+1)}, \boldsymbol{c}_{i}^{(j+1)}\right\}=\arg \min _{\alpha, \boldsymbol{B}, \boldsymbol{c}}\left\|\boldsymbol{r}_{i}^{(j+1)}(\alpha, \boldsymbol{B}, \boldsymbol{c})\right\|
$$

which extracts the mapping parameters for the $i$ th response, and for iteration $j+1$. Hence, we have the complete set of mapping parameters after $m$ PE optimizations.

\section{PRoposed SMIS ALgORITHM}

Our proposed algorithm begins with the coarse model as the initial surrogate. The algorithm incorporates explicit SM [1] and OSM [5] to speed up the convergence to the optimal solution.

Step 1) Select a coarse and fine model.

Step 2) Set $j=0$, and initialize $\boldsymbol{x}_{f}^{(0)}$. 


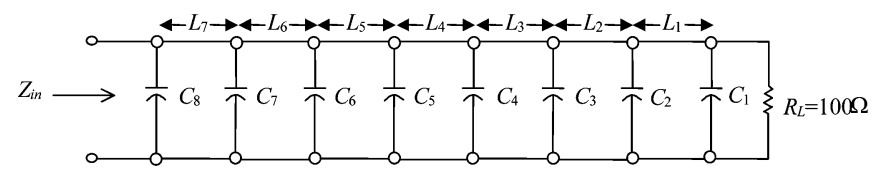

Fig. 4. Seven-section capacitively loaded impedance transformer: "Fine" model.

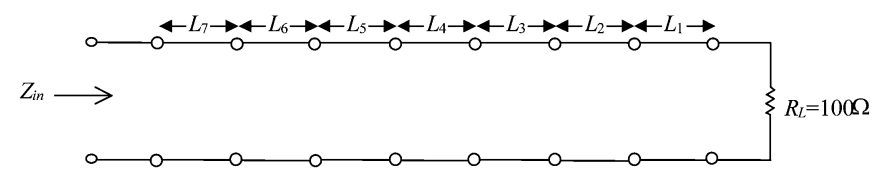

Fig. 5. Seven-section capacitively loaded impedance transformer: "Coarse" model.

TABLE I

FINE MODEL CAPACITANCES, AND THE CHARACTERISTIC IMPEDANCES FOR THE SEVEN-SECTION CAPACITIVELY LOADED IMPEDANCE TRANSFORMER

\begin{tabular}{cccc}
\hline \hline \multirow{2}{*}{ Capacitance } & Value $(\mathrm{pF})$ & Impedance & Value $(\mathrm{Ohm})$ \\
\hline$C_{1}$ & 0.025 & $Z_{1}$ & 91.9445 \\
$C_{2}$ & 0.025 & $Z_{2}$ & 85.5239 \\
$C_{3}$ & 0.025 & $Z_{3}$ & 78.1526 \\
$C_{4}$ & 0.025 & $Z_{4}$ & 70.7107 \\
$C_{5}$ & 0.025 & $Z_{5}$ & 63.9774 \\
$C_{6}$ & 0.025 & $Z_{6}$ & 58.4632 \\
$C_{7}$ & 0.025 & $Z_{7}$ & 54.3806 \\
$C_{8}$ & 0.025 & & \\
\hline \hline
\end{tabular}

Step 3) Optimize the surrogate (9) to find the next iterate $\boldsymbol{x}_{f}^{(j+1)}$ by solving $(10)$.

Step 4) Evaluate $\boldsymbol{R}_{f}\left(\boldsymbol{x}_{f}^{(j+1)}\right), \boldsymbol{J}_{f}\left(\boldsymbol{x}_{f}^{(j+1)}\right)$.

Step 5) Terminate if the stopping criteria are satisfied.

Step 6) Update the input and output mapping parameters $\alpha_{i}^{(j+1)}, \boldsymbol{B}_{i}^{(j+1)}, \boldsymbol{c}_{i}^{(j+1)}, i=1, \ldots, m$ through PE by solving (12).

Step 7) Set $j=j+1$, and go to Step 3 .

As stopping criteria for the algorithm in Step 5, the relative change in the solution vector, or the relative change in the objective function, could be employed.

\section{EXAMPLES}

\section{A. Seven-Section Capacitively Loaded Impedance Transformer}

We consider the benchmark synthetic example of a seven-section capacitively loaded impedance transformer [4]. We apply the proposed SMIS algorithm to that example. The objective function is given by $U_{f}=\max _{1 \leq i \leq m}\left|S_{11, i}\right|$. We consider a "coarse" model as an ideal seven-section transmission line (TL), where the "fine" model is a capacitively loaded TL with capacitors $C_{1, \ldots, 8}=0.025 \mathrm{pF}$. The fine and coarse models are shown in Figs. 4 and 5, respectively. Design parameters are normalized lengths $\boldsymbol{x}_{f}=\left[\begin{array}{lllllll}L_{1} & L_{2} & L_{3} & L_{4} & L_{5} & L_{6} & L_{7}\end{array}\right]^{T}$ with respect to the quarter-wave length $L_{q}$ at the center frequency of $4.35 \mathrm{GHz}$. Design specifications are

$$
\left|S_{11}\right| \leq 0.07, \quad \text { for } 1 \mathrm{GHz} \leq \omega \leq 7.7 \mathrm{GHz}
$$

with 68 points per frequency sweep. The characteristic impedances for the transformer are fixed as shown in Table I. The

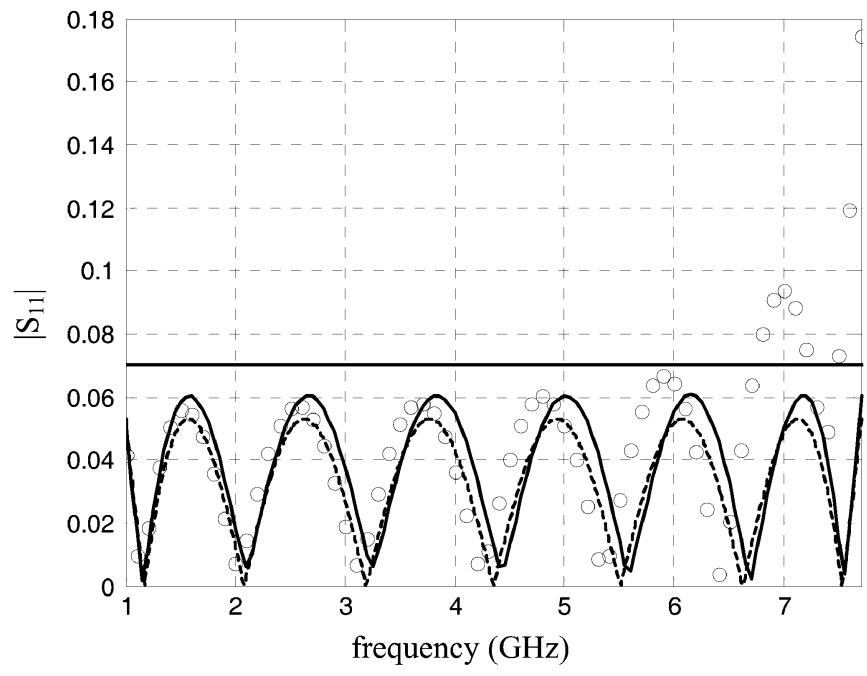

Fig. 6. Seven-section capacitively loaded impedance transformer: optimal coarse-model response (--), the optimal minimax fine-model response (-), and the fine-model response at the initial solution or at the optimal coarse-model solution (o).

TABLE II

OPtimizable Parameter Values of the SEVEn-Section CAPACITIVELY LOADED IMPEDANCE TRANSFORMER

\begin{tabular}{cccc}
\hline \hline Parameter & $\begin{array}{c}\text { Initial solution } \\
(\mathrm{m})\end{array}$ & $\begin{array}{c}\text { Solution reached } \\
\text { by the SMIS } \\
\text { algorithm } \\
(\mathrm{m})\end{array}$ & $\begin{array}{c}\text { Solution obtained } \\
\text { by direct } \\
\text { optimization } \\
(\mathrm{m})\end{array}$ \\
\hline$L_{1}$ & 0.01724138 & 0.01564205 & 0.01564205 \\
$L_{2}$ & 0.01724138 & 0.01638347 & 0.01638347 \\
$L_{3}$ & 0.01724138 & 0.01677145 & 0.01677145 \\
$L_{4}$ & 0.01724138 & 0.01697807 & 0.01697807 \\
$L_{5}$ & 0.01724138 & 0.01709879 & 0.01709879 \\
$L_{6}$ & 0.01724138 & 0.01723238 & 0.01723238 \\
$L_{7}$ & 0.01724138 & 0.01625988 & 0.01625988 \\
\hline \hline
\end{tabular}

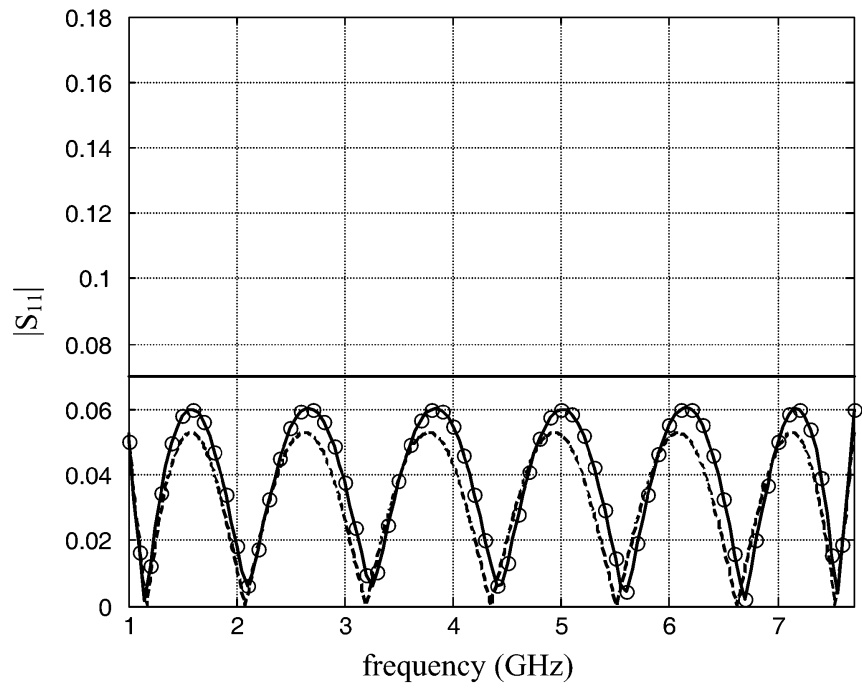

Fig. 7. Seven-section capacitively loaded impedance transformer: optimal coarse-model response (--), the optimal minimax fine-model response (-), and the fine-model response at the SMIS algorithm solution obtained after five iterations (six fine-model evaluations) (o).

Jacobians of both the coarse and fine models were obtained analytically using the adjoint network method [9]. We solve 


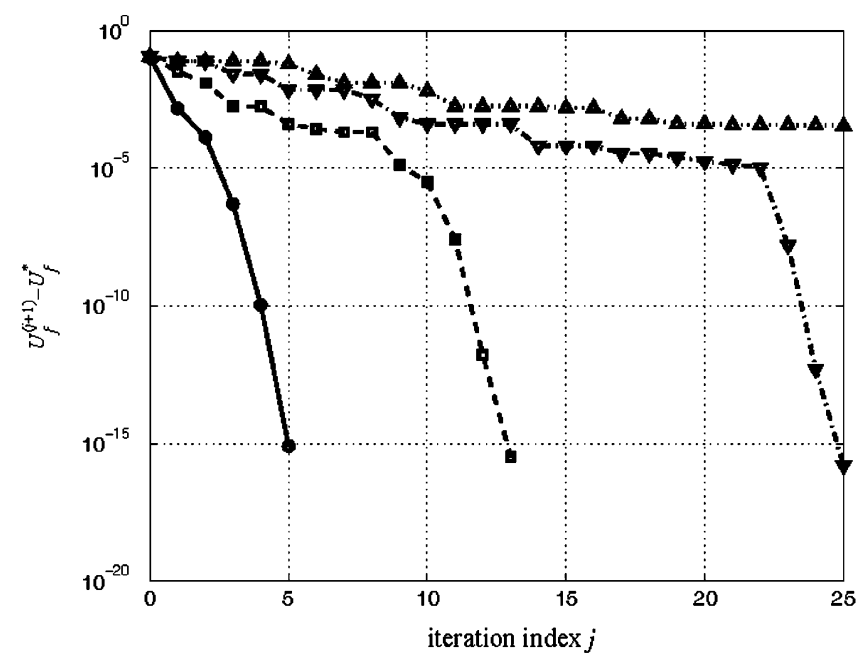

(a)

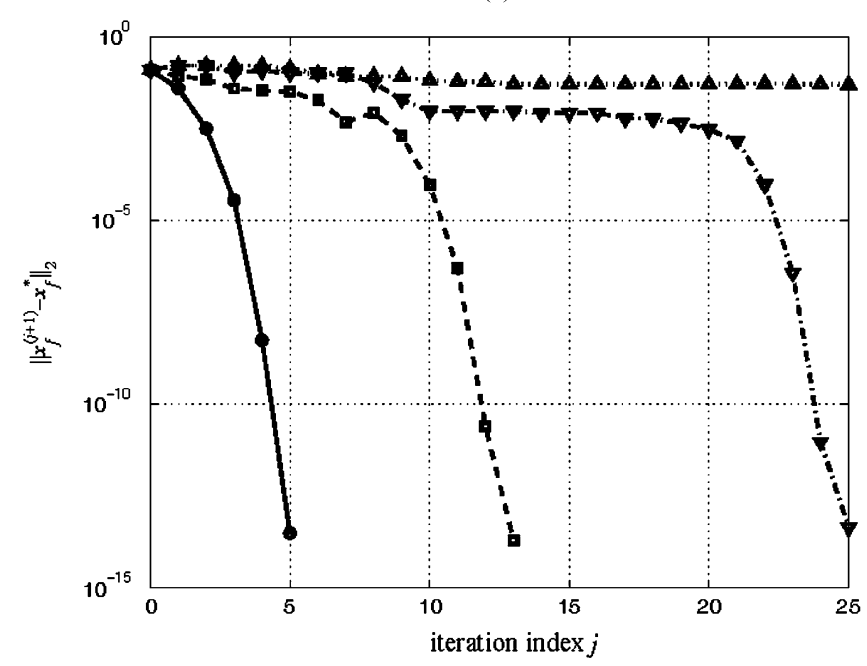

(b)

Fig. 8. (a) First 25 iterations of the difference between the fine-model objective function $U_{f}$ obtained using the SMIS algorithm (o) and the fine-model objective function at the fine-model minimax solution $U_{f}^{*}$ obtained by the Hald-Madsen algorithm ( $\square$ ), the HASM surrogate optimization algorithm using exact gradients $(\nabla)$, and the HASM surrogate optimization algorithm using the Broyden update $(\Delta)$. (b) The corresponding difference between the designs.

the PE problem using the Levenberg-Marquardt algorithm for nonlinear least squares optimization available in the MATLAB Optimization Toolbox. ${ }^{1}$

Optimizing the fine model directly using the gradient-based minimax method of Madsen [13], and Hald and Madsen [14] confirms that the problem has numerous local solutions. Starting from the optimal coarse-model solution (the initial solution for the SMIS method), the Hald-Madsen minimax algorithm needs 13 iterations, or 13 fine-model evaluations, to converge to the fine-model minimax solution. Note that both the direct optimization method of Hald and Madsen and the SMIS approach employ exact gradients.

The fine-model response at the optimal coarse-model solution is shown in Fig. 6. Table II shows the lengths for solutions obtained using the SMIS algorithm and the fine-model direct minimax optimization solution [13], [14]. Our SMIS algorithm

${ }^{1}$ MATLAB, ver. 6.1, MathWorks Inc., Natick, MA, 2001.

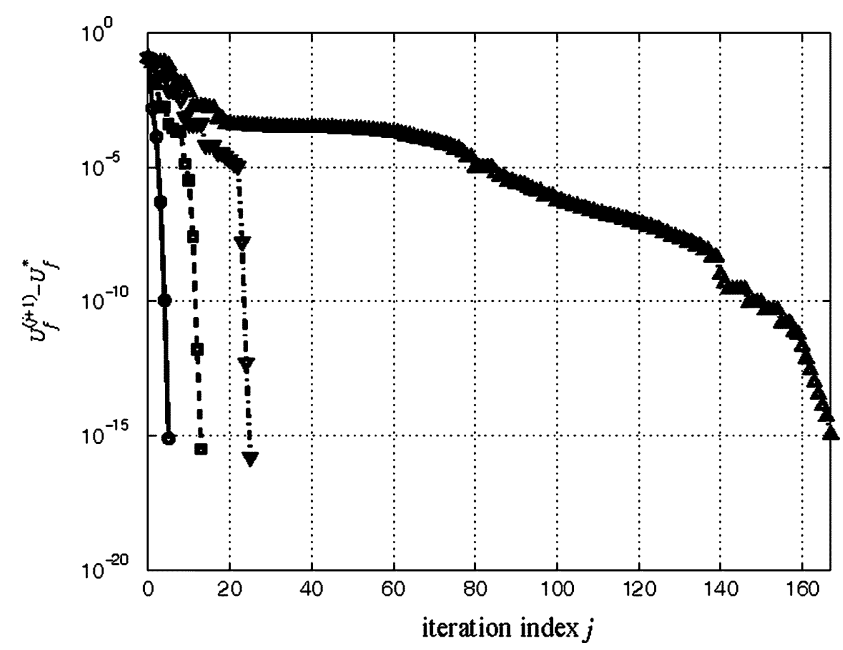

(a)

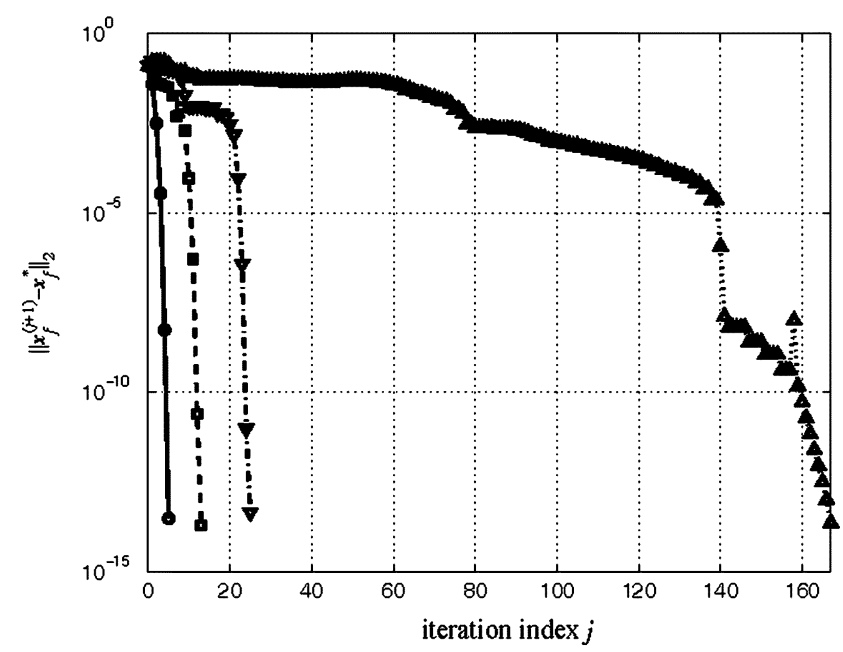

(b)

Fig. 9. (a) Difference between the fine-model objective function $U_{f}$ obtained using the SMIS algorithm $(0)$ and the fine-model objective function at the fine-model minimax solution $U_{f}^{*}$ obtained by the Hald-Madsen algorithm ( $\square$ ), the HASM surrogate optimization algorithm using exact gradients $(\nabla)$, and the HASM surrogate optimization algorithm using the Broyden update $(\Delta)$. (b) The corresponding difference between the designs.

took six fine-model evaluations or five iterations to reach the same accurate solution as the Hald-Madsen direct minimax optimization algorithm.

Fig. 7 shows the fine-model response at the SMIS algorithm solution. The difference between the minimax objective function at the optimal minimax fine-model response and the response obtained using the SMIS algorithm is shown in Figs. 8 and 9.

Corresponding results reached by the Hald-Madsen method are shown in Figs. 8 and 9. In these figures, we show the HASM surrogate exploiting exact gradients. The minimax objective function and solution reached by the HASM surrogate optimization approach using the Broyden update [10] are also shown. The four methods converged to the same highly accurate solution.

The optimization methods used for solving (1) and a comparison is shown in Table III. Using the adjoint technique, the SMIS algorithm was able to obtain the same optimum solution as the Hald-Madsen algorithm within an error of $10^{-15}$ after only five iterations. 
TABLE III

Optimization METHODS USED ON THE SEVEN-SECTION CAPACITIVELY LOADED IMPEDANCE TRANSFORMER

\begin{tabular}{cccc}
\hline \hline \multirow{2}{*}{ Problem } & Method & $\begin{array}{c}\text { Number of } \\
\text { Iterations }\end{array}$ & $\begin{array}{c}\text { Number of } \\
\text { fine model } \\
\text { evaluations }\end{array}$ \\
\hline & fminimax* & 14 & 153 \\
\cline { 2 - 4 }$(1)$ & HASM & 25 & 26 \\
\cline { 2 - 4 } & Hald-Madsen & 13 & 13 \\
\cline { 2 - 4 } & SMIS & 5 & 6 \\
\hline \hline
\end{tabular}

* The fminimax routine available in the Matlab Optimization Toolbox [13].

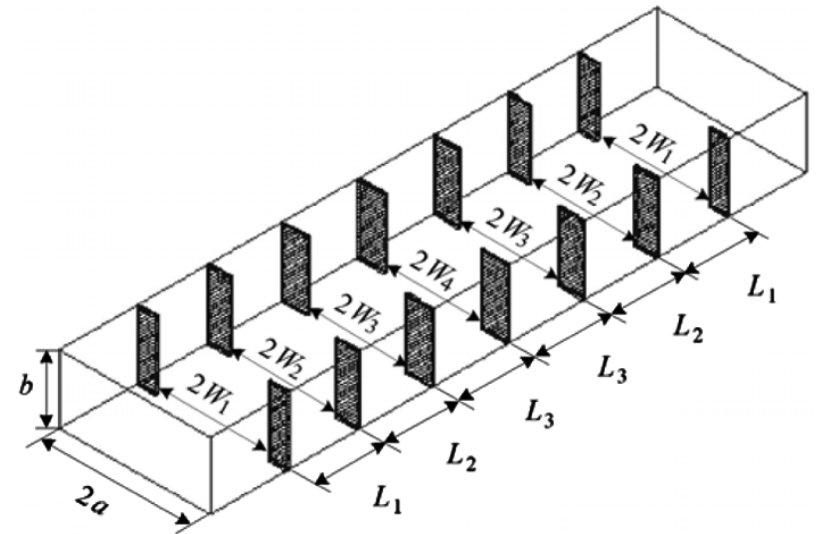

(a)

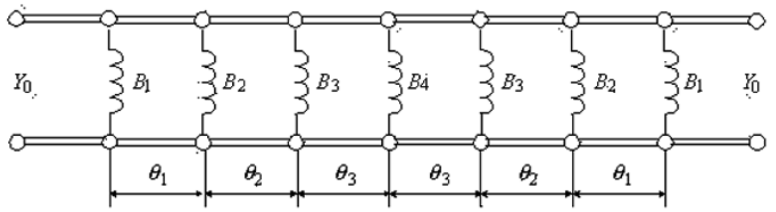

(b)

Fig. 10. Six-section $H$-plane waveguide filter [7]. (a) Physical structure. (b) Coarse model as implemented in MATLAB .

In contrast to SMIS, the standard minimax optimizer available in MATLAB was able to reach the same optimum direct optimization result in 14 iterations (153 fine-model evaluations), while the Hald-Madsen algorithm reached the optimum finemodel solution in 13 iterations (13 fine-model evaluations). The HASM algorithm exploiting exact gradients took 25 iterations (26 fine-model evaluations) to reach the optimum fine-model solution to the same error of $10^{-15}$.

The Hald-Madsen algorithm exploits sequential linear programming (SLP) using trust regions, combined with a Newton iteration. The MATLAB minimizer (fminimax) exploits a sequential quadratic programming (SQP) method with line searches.

\section{B. Six-Section H-Plane Waveguide Filter}

The physical structure of the six-section $H$-plane waveguide filter is shown in Fig. 10(a) [7]. We simulate the fine model using Agilent High Frequency Structure Simulator (HFSS). ${ }^{2}$ The design parameters are the lengths and widths, namely,

$$
\boldsymbol{x}_{f}=\left[\begin{array}{lllllll}
L_{1} & L_{2} & L_{3} & W_{1} & W_{2} & W_{3} & W_{4}
\end{array}\right]^{T} .
$$

${ }^{2}$ Agilent HFSS, ver. 5.6, HP EESof, Agilent Technol., Santa Rosa, CA, 2000.

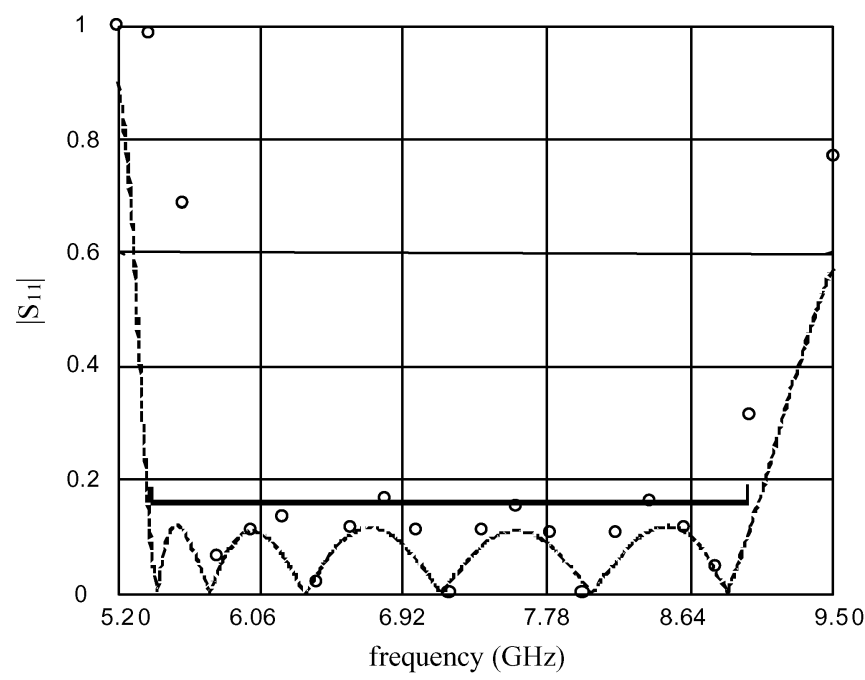

(a)

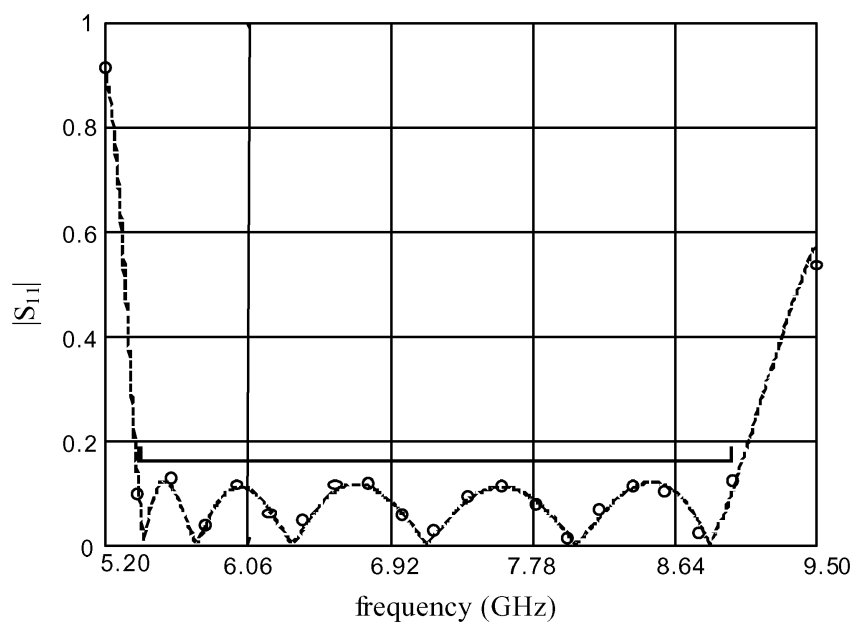

(b)

Fig. 11. $H$-plane filter optimal coarse-model response (-), and the HFSS (fine-model) response: (a) at the initial solution (o) and (b) at the SMIS algorithm solution reached after three iterations $(0)$.

TABLE IV

OPTIMIZABLE PARAMETER VALUES OF THE SIX-SECTION $H$-Plane WAVEGUIDE FILTER

\begin{tabular}{ccc}
\hline \hline Parameter & $\begin{array}{c}\text { Initial solution } \\
\text { (inches) }\end{array}$ & $\begin{array}{c}\text { Solution reached by the } \\
\text { SMIS algorithm } \\
\text { (inches) }\end{array}$ \\
\hline$W_{1}$ & 0.48583 & 0.51397 \\
$W_{2}$ & 0.43494 & 0.47244 \\
$W_{3}$ & 0.40433 & 0.44501 \\
$W_{4}$ & 0.39796 & 0.44627 \\
$L_{1}$ & 0.65585 & 0.63142 \\
$L_{2}$ & 0.65923 & 0.63922 \\
$L_{3}$ & 0.67666 & 0.65705 \\
\hline \hline
\end{tabular}

Design specifications are

$$
\begin{aligned}
& \left|S_{11}\right| \leq 0.16, \quad \text { for } 5.4 \mathrm{GHz} \leq \omega \leq 9.0 \mathrm{GHz} \\
& \left|S_{11}\right| \geq 0.85, \quad \text { for } \omega \leq 5.2 \mathrm{GHz} \\
& \left|S_{11}\right| \geq 0.5, \quad \text { for } \omega \geq 9.5 \mathrm{GHz}
\end{aligned}
$$

with 23 points per frequency sweep.

A waveguide with a cross section of 1.372 in $\times 0.622$ in $(3.485 \mathrm{~cm} \times 1.58 \mathrm{~cm})$ is used. The six sections are separated by 
seven $H$-plane septa, which have a finite thickness of 0.02 in $(0.508 \mathrm{~mm})$. The coarse model consists of lumped inductances and dispersive TL sections [see Fig. 10(b)]. There are various approaches to calculate the equivalent inductive susceptance of an $H$-plane septum. We use a simplified version of a formula due to Marcuvitz [15]. The coarse model is simulated using MATLAB . The fine model exploits the Agilent HFSS simulator. One frequency sweep takes $2.5 \mathrm{~min}$ on an Intel Pentium 4 (3 GHz) machine with 1-GB RAM and running in Windows XP Pro. Seven fine-model simulations, due to the seven 0.01-in perturbations, are required to find the fine-model Jacobian offline using the finite-difference method. Thus, the time taken for fine model and Jacobian calculation is $21 \mathrm{~min} /$ iteration on an Intel P4 machine. Fig. 11(a) shows the fine-model response at the initial solution. Fig. 11(b) shows the fine-model response after running our SMIS algorithm using HFSS. The total time taken was 126 min on an Intel P4 3-GHz machine. Table IV shows the initial and optimal design parameter values of the six-section $H$-plane waveguide filter.

\section{CONCLUSION}

We have presented a powerful algorithm based on a novel SMIS framework that delivers the solution accuracy expected from direct gradient-based optimization using SLP, yet converges in a handful of iterations. It aims at matching a surrogate (mapped coarse model) with the fine model within a local region of interest by introducing more degrees of freedom into the SM. Convergence is demonstrated through a seven-section capacitively loaded impedance transformer. We compare the SMIS algorithm with major direct minimax optimization algorithms. It yields the same solution within an error of $10^{-15}$ as the Hald-Madsen algorithm. A highly optimized $H$-plane filter design emerges after only four EM simulations (three iterations), excluding necessary Jacobian estimations, using the new algorithm with sparse frequency sweeps.

\section{ACKNOWLEDGMENT}

The authors thank Agilent Technologies, Santa Rosa, CA, for making Agilent HFSS available. The assistance of Q. S. Cheng, S. A. Dakroury, S. Koziel, A. S. Mohamed, and N. K. Nikolova, all of McMaster University, Hamilton, ON, Canada, is also gratefully acknowledged.

\section{REFERENCES}

[1] J. W. Bandler, R. M. Biernacki, S. H. Chen, P. A. Grobelny, and R. H. Hemmers, "Space mapping technique for electromagnetic optimization," IEEE Trans. Microwave Theory Tech., vol. 42, pp. 2536-2544, Dec. 1994.

[2] J. W. Bandler, Q. Cheng, S. A. Dakroury, A. S. Mohamed, M. H. Bakr, K. Madsen, and J. Søndergaard, "Space mapping: The state of the art," IEEE Trans. Microwave Theory Tech., vol. 52, pp. 337-361, Jan. 2004.

[3] J. W. Bandler, Q. S. Cheng, N. K. Nikolova, and M. A. Ismail, "Implicit space mapping optimization exploiting preassigned parameters," IEEE Trans. Microwave Theory Tech., vol. 52, pp. 378-385, Jan. 2004.

[4] M. H. Bakr, J. W. Bandler, K. Madsen, and J. Søndergaard, "An introduction to the space mapping technique," Optimization Eng., vol. 2, pp. 369-384, 2001.

[5] J. W. Bandler, Q. S. Cheng, D. H. Gebre-Mariam, K. Madsen, F. Pedersen, and J. Søndergaard, "EM-based surrogate modeling and design exploiting implicit, frequency and output space mappings," in IEEE MTT-S Int. Microwave Symp. Dig., Philadelphia, PA, 2003, pp. 1003-1006.
[6] J. W. Bandler, Q. S. Cheng, S. A. Dakroury, D. M. Hailu, K. Madsen, A. S. Mohamed, and F. Pedersen, "Space mapping interpolating surrogates for highly optimized EM-based design of microwave devices," in IEEE MTT-S Int. Microwave Symp. Dig., Fort Worth, TX, 2004, pp. $1565-1568$.

[7] G. L. Matthaei, L. Young, and E. M. T. Jones, Microwave Filters, Impedance-Matching Networks, and Coupling Structures, $1 \mathrm{st}$ ed. New York: McGraw-Hill, 1964, pp. 521-582.

[8] J. E. Dennis, Jr., private communication, 2002.

[9] J. W. Bandler and R. E. Seviora, "Computation of sensitivities for noncommensurate networks," IEEE Trans. Circuit Theory, vol. CT-18, pp. 174-178, Jan. 1971.

[10] M. H. Bakr, J. W. Bandler, K. Madsen, J. E. Rayas-Sánchez, and J. Søndergaard, "Space mapping optimization of microwave circuits exploiting surrogate models," IEEE Trans. Microwave Theory Tech., vol. 48, pp. 2297-2306, Dec. 2000.

[11] K. Madsen and J. Søndergaard, "Convergence of hybrid space mapping algorithms," Optimization Eng., vol. 5.2, pp. 145-156, 2004.

[12] N. M. Alexandrov, J. E. Dennis, Jr., R. M. Lewis, and V. Torczon, “A trust-region framework for managing the use of approximation models in optimization," Structural Optimization, vol. 15, pp. 16-23, 1998.

[13] K. Madsen, "An algorithm for minimax solution of overdetermined systems of nonlinear equations," J. Inst. Math. Applicat., vol. 16, pp. 321-328, 1975.

[14] J. Hald and K. Madsen, "Combined LP and quasi-Newton methods for minimax optimization," Math. Programming, vol. 20, pp. 49-62, 1981.

[15] N. Marcuvitz, Waveguide Handbook, 1st ed. New York: McGraw-Hill, 1951, p. 221.

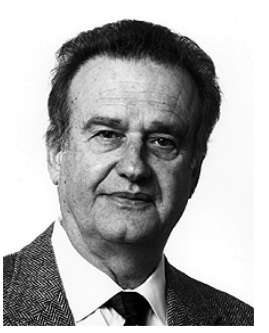

John W. Bandler (S'66-M'66-SM'74-F'78) was born in Jerusalem, on November 9, 1941. He studied at Imperial College of Science and Technology, London, U.K., from 1960 to 1966 . He received the B.Sc. (Eng.), Ph.D., and D.Sc. (Eng.) degrees from the University of London, London, U.K., in 1963, 1967, and 1976, respectively.

In 1966, he joined Mullard Research Laboratories, Redhill, Surrey, U.K. From 1967 to 1969, he was a Post-Doctorate Fellow and Sessional Lecturer with the University of Manitoba, Winnipeg, MB, Canada. In 1969, he joined McMaster University, Hamilton, ON, Canada, where he has served as Chairman of the Department of Electrical Engineering and Dean of the Faculty of Engineering. He is currently Professor Emeritus in Electrical and Computer Engineering, and directs research in the Simulation Optimization Systems Research Laboratory. He was President of Optimization Systems Associates Inc. (OSA), which he founded in 1983, until November 20, 1997, the date of acquisition of OSA by the Hewlett-Packard Company (HP). OSA implemented a first-generation yield-driven microwave CAD capability for Raytheon in 1985, followed by further innovations in linear and nonlinear microwave CAD technology for the Raytheon/Texas Instruments Joint Venture MIMIC Program. OSA introduced the computer-aided engineering (CAE) systems RoMPE in 1988, HarPE in 1989, OSA90 and OSA90/hope in 1991, Empipe in 1992, and Empipe3D and EmpipeExpress in 1996. OSA created empath in 1996, marketed by Sonnet Software Inc. He is currently President of Bandler Corporation, Dundas, ON, Canada, which he founded in 1997. He has authored or coauthored over 365 papers from 1965 to 2004. He contributed to Modern Filter Theory and Design (New York: Wiley-Interscience, 1973) and Analog Methods for Computer-aided Analysis and Diagnosis (New York: Marcel Dekker, 1988). Four of his papers have been reprinted in Computer-Aided Filter Design (New York: IEEE Press, 1973), one in each of Microwave Integrated Circuits (Norwood, MA: Artech House, 1975), Low-Noise Microwave Transistors and Amplifiers (New York: IEEE Press, 1981), Microwave Integrated Circuits, 2nd ed.(Norwood, MA: Artech House, 1985), Statistical Design of Integrated Circuits (New York: IEEE Press, 1987), and Analog Fault Diagnosis (New York: IEEE Press, 1987). He joined the Editorial Boards of the International Journal of Numerical Modeling (1987), the International Journal of Microwave and Millimeterwave Computer-Aided Engineering (1989), and Optimization Eng. in 1998. He was Guest Editor of the International Journal of Microwave and Millimeter-Wave Computer-Aided Engineering Special Issue on Optimization-Oriented Microwave CAD (1997). He was Guest Co-Editor of the Optimization Eng. Special Issue on Surrogate Modelling and Space Mapping for Engineering Optimization (2001). 
Dr. Bandler is a Fellow of the Canadian Academy of Engineering, the Royal Society of Canada, the Institution of Electrical Engineers (U.K.), and the Engineering Institute of Canada. He is a member of the Association of Professional Engineers of the Province of Ontario (Canada) and a member of the Massachusetts Institute of Technology (MIT) Electromagnetics Academy. He was an associate editor of the IEEE TRANSACTIONS ON MICROWAVE THEORY AND TECHNIQUES (1969-1974), and has continued serving as a member of the Editorial Board. He was guest editor of the IEEE TRANSACTIONS ON Microwave Theory and TeChNiQues Special Issue on Computer-Oriented Microwave Practices (1974) and guest co-editor of the IEEE TRANSACTIONS ON MicrowaVE THEORY AND TECHNIQUES Special Issue on Process-Oriented Microwave CAD and Modeling (1992). He was guest editor of the IEEE TRANSACTIONS ON MicrowaVe THEORY AND TeChNIQUES Special Issue on Automated Circuit Design Using Electromagnetic Simulators (1997). He is guest co-editor of the IEEE TRANSACTIONS ON MiCROWAVE THEORY AND TECHNIQUES Special Issue on Electromagnetics-Based Optimization of Microwave Components and Circuits (2004). He has served as chair of the MTT-1 Technical Committee on Computer-Aided Design. He was the recipient of the 1994 Automatic Radio Frequency Techniques Group (ARFTG) Automated Measurements Career Award and the 2004 Microwave Application Award presented by the IEEE MTT-S.

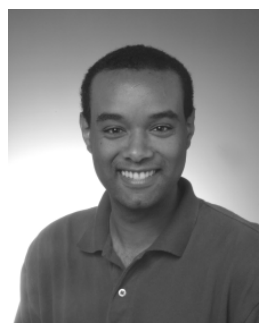

Daniel M. Hailu (S'99) was born in Winnipeg, MB, Canada, in 1979. He received the B.Eng. degree (with distinction) in computer engineering from McMaster University, Hamilton, ON, Canada, in 2002.

In May 2002, he joined the Simulation Optimization Systems Research Laboratory and the Department of Electrical and Computer Engineering, McMaster University, as a graduate student. His research interests are in CAD and modeling of microwave circuits, EM optimization, SM technology, OSM, device modeling, and CAD methods for antennas

Mr. Hailu was the recipient of the 2001-2002 Dr. Rudolf de Buda Scholarship for academic achievement and the 2002-2003 Natural Sciences and Engineering Research Council Undergraduate Student Research Award (NSERC USRA).

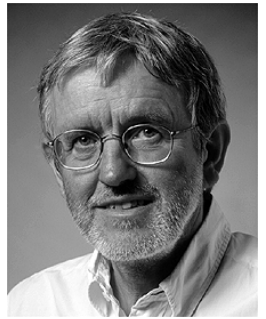

Kaj Madsen was born in Hjørring, Denmark, in 1943. He received the Cand.Scient. degree in mathematics from the University of Aarhus, Aarhus, Denmark, in 1968, and the Dr.Techn. degree from the Technical University of Denmark (DTU), Lyngby, Denmark, in 1986

From 1968 to 1988, he was a Lecturer of numerical analysis (aside from the 1973-1974 academic year, when he was with AERE Harwell, Didcot, U.K.). Most of his career has been with the Department for Numerical Analysis, DTU. From 1981 to 1983, he was with the Computer Science Department, Copenhagen University. During the summer of 1978, he visited McMaster University, Hamilton, ON, Canada. In 1988, he became a Full Professor. In 1993, he joined the Department of Mathematical Modelling, DTU, and during 1995-2000, he was Head of that department. In 2000, he took an active part in forming the new Department of Informatics and Mathematical Modelling, DTU, which includes computer science and applied mathematics, and since January 2001, he has headed that department. His primary fields of interest in teaching and research are nonlinear optimization including SM techniques and global optimization, and validated computing using interval analysis.

Dr. Madsen arranged several international workshops on linear programming, parallel algorithms, and surrogate optimization during the 1990s.

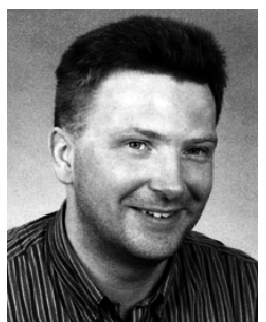

Frank Pedersen was born in Roskilde, Denmark, in 1968. He received the M.Sc. degree in engineering from the Technical University of Denmark, Lyngby, Denmark, in 2001, and is currently working toward the Ph.D. degree in engineering at the Technical University of Denmark.

During 2002, he was a Research Assistant with the Technical University of Denmark. His research concerns the development of nonlinear optimization techniques for design and control problems. 